\title{
The Application Research on the Computer Virtual Technology and Modeling Technology in the Sports Movement Simulation
}

\author{
Guo sheng \\ P.E.College, Beihua University \\ Jilin City, Jilin Province, China
}

Keywords: virtual technology; modeling technology; sports; the simulation

\begin{abstract}
In this paper, the virtual reality technology how used in the simulation system is studied, of which the practicality and limitation is analyzed. Graphics modeling technology used in the simulation of the system is regarded as a starting point and a combining point. The author expounds the theoretical basis and key technology of the graphical modeling and virtual reality technology application in sport simulation, and describes the sports technology of joint chain model on human body levels. In graphics modeling, the virtual reality technology is organically combined to realize the simulation scene demonstration system based on virtual reality modeling language VRML. The computer virtual technology and modeling technology can be used in the further research on athlete actions and help the quantitative analysis of specific actions, having the important meaning for improving the training methods.
\end{abstract}

\section{Introduction}

Using graphical modeling technology not only can describe the complex logical relationship between objects in the system, but also the corresponding relationship between icon and algorithm of the algorithms library. During the modeling, the icon call way of the graphical environment of the front desk can complete the algorithm calls of the back desk. Therefore, it is convenient to obtain and express modeling information visualization. Virtual reality is a science including the person and the information, whose essence is to use computer to enforce comprehensive simulation of reality technology or fictional world to create a lifelike $3 \mathrm{~d}$, that is, the integration of sight, hearing, and touch feeling. Virtual reality technology is a comprehensive technology, which consists of computer graphics techniques, man-machine interface technology, multimedia technology, stereo vision and artificial intelligence and other aspects of the technology. From technology application perspective, the system simulation technology is a comprehensive technology, based on similar principles, system technology, control theory, computer technology, information technology and professional technology in its application fields, using mathematical model or some material objects to make dynamic experimental study on the reality system or illusion system with uncertainty factors.

\section{The technical base of graphical modeling in the virtual simulation system}

The graphical modeling system can provide customers with the graphical modeling environment with a good interface, the complete function and simple operation. The virtual simulation system can show the dynamic and interactive $3 \mathrm{~d}$ virtual scene model to users' target system.

MVC design mode.MVC (Model-View-Controller) is a kind of interface of organization structures. Model closes the core data of the problem and the calculation relationship of the logic function, and provides the operation process of completing treatment. View shows the model data 
and its logical relationship and state information to users in particular ways. Controller is to handle interactive operation between users and software and can control the spread of any changes in the model, to ensure the corresponding relationship between the user interface and the model.

ActiveX control technology. ActiveX is to update and upgrade the OLE control technology widely applied, whose essence is to realize high application compatibility following certain standards. OLE also provides the reusable software development standards based on components, which can use the already made component parts to build applications.

Virtual reality modeling language (VRML). VRML basic working principle can be generalized as follows: text description, remote transmission and the local computer generated. The basic characteristics of VRML can be summarized as: household output-related, low bandwidth feasibility based on the ASCII, real-time 3 D coloring engine and its extensibility.

\section{The technology realization of the graphical modeling system}

The graphical modeling system adopts the document of the MVC design model, and forms the application program framework based on MFC library, as shown in figure 1.

The application program of CGEngineerApp keeps and maintains a pointer list of all the document template. It is a list of the structure. Application program distributes a CMultiDoc Template object to each document type dynamic. Function prototypes for:

CMultiDocTemplate (UINTnIDResource, CRuntimeClass * pDocClass, CRuntimeClass * pFrameClass, CRuntimeClass * pViewClass);

By calling CFrameWnd: : GetActiveDocument, MDI frame window can obtain a pointer to the child window including multiple files interface from MDI frame window.

CMDIChildWnd calls GetMDIFrame to get MDI frame window (CMDIFrameWnd).

CWinApp calls AfxGetMainWnd to a pointer to the activity main window of the application program.

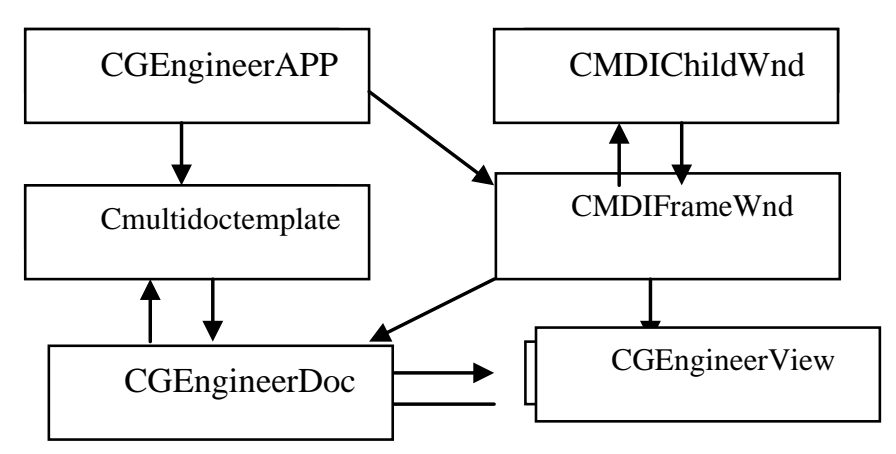

Figure 1. The class structure and relationship of the system framework

In the program design of the MFC, the system data can be saved by using document class. Following this principle, the system stores and maintains a list of the graphic objects in CGEngineerDoc document class. When the users set up icon or build simulation model in the working area, each primitive or icon drawn in work area will be added to the list of the graphic objects. View is a visual performance of the data stored in a document, and can draw the graphics object stored in its list of the document in the user work area by using the function OnDraw (CDC * PDC) in CGEngineerView.

The lists of graphic objects are as follows:

typedefCTypedPtrList < CObList, CDrawObj * > CDrawObjList:

CDrawObjList * m_pObjects; 


\section{The technical realization of the virtual simulation scene demonstration system}

After building the systemic graphic simulation model in the graphical modeling system, users can produce a $3 \mathrm{~d}$ virtual simulation scene on the base of VRML virtual corresponding to the simulation model by using the virtual simulation scene demonstration system. In the formation process, all sorts of modeling nodes, sensor node and other nodes with special functions of VRML are involved. According to the operation data of the simulation model, the virtual object of virtual scene can adjust its real-time state in order to react the real dynamic change of the equipment state in the system.

Making virtual equipment by using 3 DSMAX. 3 DSMAX provides dynamics modeling after opening 3 DSMAX to create a new model. It is necessary to establish a viewpoint because 3 DSMAX does not match VRML modeling unit. We can't get the right perspective if the camera 3 DSMAX scene doesn't directly export VRML. On the base of $3 \mathrm{~d}$ virtual equipment, we can choose 3 DSMAX to export menu options. In the pop-up Exporter dialog, corresponding parameters should be set, and the system can export $3 \mathrm{~d}$ model made by 3 DSMAX into VRML.

Controlling simulation picture by using the time sensor node. Using the time sensor node (TimeSensor) to drive continuous simulation pictures refreshes data. TimeSensor node is a clock. It can produce relevant time events with the passage of time, using these events to control some behaviors relevant to time. When TimeSensor is in an open position (enabled = TRUE), and the time is a specified value in startTime, it begins to develop events and output the continuous events time. Event time intervals produced from TimeSensor can be controlled by using cycInterval domain

The grammar of TimeSensor node is as follows:

TimeSensor \{

EventOut SFBool isActive

EventOut SFTime time

EventOut SFTime cycleTime

EventOut SFFloat fraction_changed

ExposedField SFTime cycleInterval 1.0

ExposedField SFBool enabled TRUE

ExposedField SFTime startTime 0

ExposedField SFTime stopTime 0

ExposedField SFBool loop FALSE

\}

Feeling user action by using touch sensor node. Touchsensor provides users a means of interaction of the VRML world. We can use it to perceive the moving or click action of the mouse of the user on the nodes in the group. In the simulation system, the changes of opening and closing in this system can be controlled by defining touch sensor node on the nodes in the group of the virtual equipment. The grammar of Touchsensor node is as follows:

Touchsensor \{

EventOut SFBool isOver

EventOut SFVec3f hitPoint_changed

EventOut SFVec3f hitNormal_changed

EventOut SFVec3f hitTexCoord_changed

EventOut SFBool isActive

EventOut SFTime touchTime

ExposedField SFBool TRUE

\}

Controlling virtual equipment position by dragging sensor nodes. When users establish graphic simulation models, according to the equipment icon of them, the system will find corresponding virtual equipment in $3 \mathrm{~d}$ virtual equipment library, and according to $3 \mathrm{~d}$ space position of each virtual equipment stored in space database, the system will generate the corresponding virtual scene. The space position stored in $3 \mathrm{~d}$ spatial database tacitly approves $2 \mathrm{~d}$ 
position plane stored in graphical modeling environment. Although the user can give other definitions in modeling process, but he/she is likely to be unsatisfied with the virtual equipment spatial position generated in a virtual scene. At this time, this system allows users to adjust it by dragging sensor nodes in VRML. There are three types of drag sensor nodes: Plane sensor, allowing users to move objects along XY plane ; Cylinder sensor, taking the motion mapping to a virtual cylinder; Spherical sensor, taking the motion mapping to a virtual sphere.

\section{Summary}

According to the built model in the graphical modeling in the virtual simulation scene demonstration system and the three dimensional space information, the corresponding real-time $3 \mathrm{~d}$ model can be generated in the process of formation. Further studying the action in each link of athlete technology helps the quantitative analysis. Combining with human physiology and the principle of sports biomechanics, we will improve training methods to make the sports training not depended on experience and into a theorized, digital age.

\section{References}

[1] Zhang Yujin, “Image processing and analysis”, tsinghua university press, 1999.

[2] Xu Xiaogang, Yu Jinhui, Ma Lizhuang, “Complex object contour extraction”, Chinese journal of image and graphics.

[3] Huang Xinxin, Qi Deyu, Wang Xiuyuan, “ The research on extraction algorithms of real-time image contour”, computer research. 2000

[4] Chen Jiashi, “3 d reconstruction movement under the perspective projection”, computer aided design and graphics journals, 1999.

[5] Wang Rencheng, Huang Changhua, Wang Jijun, Jin Dewen, "Image processing based on the index point of the camera in the human motion analysis system”, tsinghua university press, 1999.

[6] Li Yang "the sport balance function evaluation research progress". Shenyang Sorts institude jurnal.

[7] Li Wenbin, Wang Deming. “The sport balance function test system research progress”. The human Ergonomic.

[8] Zhang Xin “The sport balance function evaluation research progress”"Phisical medical learn to learn and rehabilitation.

[9] 6. JinMin, xu punctual. The same moment in the application of pattern recognition. The computer engineering and application. 2004

[10] 7. WangBin. Constraint sequence of target motion detection and tracking the research problem. Northwest university press. 2004 